\title{
Flippable Pairs and Subset Comparisons in Comparative Probability Orderings
}

\author{
ROBIN CHRISTIAN \\ Deptartment of Combinatorics and Optimization, University of Waterloo \\ r3christ@math. uwaterloo.ca \\ Marston Conder 1 \\ Department of Mathematics, University of Auckland \\ m. conder@auckland.ac.nz \\ ARKADII SLINKd 2 \\ Department of Mathematics, University of Auckland \\ a.slinko@auckland.ac.nz
}

\begin{abstract}
We show that every additively representable comparative probability order on $n$ atoms is determined by at least $n-1$ binary subset comparisons. We show that there are many orders of this kind, not just the lexicographic order. These results provide answers to two questions of Fishburn et al (2002). We also study the flip relation on the class of all comparative probability orders introduced by Maclagan. We generalise an important theorem of Fishburn, Pekeč and Reeds, by showing that in any minimal set of comparisons that determine a comparative probability order, all comparisons are flippable. By calculating the characteristics of the flip relation for $n=6$ we discover that the polytopes associated with the regions in the corresponding hyperplane arrangement can have no more than 13 facets and that there are 20 regions whose associated polytopes have 13 facets. All the neighbours of the 20 comparative probability orders which correspond to those regions are representable.
\end{abstract}

AMS classification: 60A05, 91B08 (primary), 06A07, 91A12, 91E99 (secondary)

Keywords: comparative probability, flip relation, elicitation, subset comparisons, additively representable linear orders

\footnotetext{
${ }^{1}$ Research partially supported by the N.Z. Centres of Research Excellence Fund (grant UOA 201).

${ }^{2}$ Corresponding author
} 


\section{Introduction}

In this paper we study algebraic and combinatorial properties of comparative probability orders on a finite set $X$ of $n$ elements. A significant part of this research direction was initiated by Fishburn et al [9] and is related to problems in decision theory and the theory of measurement, especially to the problem of preference elicitation. We answer two questions posed in that paper.

Another factor that influenced our research was a paper by Maclagan [11, who introduced the concept of a flippable pair, and related comparative probability orders to oriented matroids and the Baues problem. We believe that the concept of a flippable pair is very important and adds richness to the whole theory. Maclagan also formulated a number of very interesting questions (see [11, p. 295]). We develop the concept of a lexicographic product of two orders, as briefly outlined by Maclagan (see [11 p. 294]), and use it extensively throughout this paper. In particular we give a new method of constructing comparative probability orders which are not even 'almost representable' by a probability measure. Only one such example appears to have been given previously in the literature [10.

Fishburn et al [9] paid significant attention to elicitation of comparative probability orders representable by a probability measure. They proved that any minimal set of comparisons that determines an order $\preceq$ on $2^{X}$ consists of critical pairs. By relating flippable pairs with irreducible elements of the discrete cone corresponding to $\preceq$, we prove a much stronger condition, namely that any minimal set of comparisons consists of flippable pairs. For orders which are not representable by probability measures, we show that the sequence of flippable pairs does not determine the order uniquely. For $n=6$ we discovered eight pairs of comparative probability orders with the same sequences of flippable pairs.

Let $\mathcal{L}_{n}$ be the set of all comparative probability orders on the set consisting of $n$ elements which are representable by an order preserving positive probability measure. Fishburn et al formulated the following two problems (see [9] p. 243, Open problems 2 and 3]):

1. Show that no order $\preceq \in \mathcal{L}_{n}$ is determined by $n-2$ (or fewer) binary subset comparisons;

2. Decide whether every $\preceq \in \mathcal{L}_{n}$ which is determined by $n-1$ comparisons has the structure of the lexicographic order determined by $\left\{i_{1}, \ldots, i_{j}\right\} \prec i_{j+1}$ for $j=1,2, \ldots, n-1$.

We prove the first statement in Section [5] and show that the lexicographic order is just one of many orders that can be determined by $n-1$ comparisons. We cannot characterise all of these orders, but we can show that they are in one-to-one correspondence with the comparative probability orders on the set consisting of $n-1$ elements determined by exactly $n-1$ comparisons.

It is a well-known fact that every representable comparative probability order on $n$ elements can be characterised by a region in the hyperplane arrangement $\mathcal{H}_{n}[5]$. The closure of every such region is a convex polytope. Maclagan [11 asked how many facets those polytopes have. We fully answer this question for $n=6$ in Section 8

Some of these results were delivered at the 4th International Symposium on Imprecise Probabilities and Their Applications (ISIPTA 05); see [2].

\section{Preliminaries}

Definition 1. Let $X$ be a finite set. Any reflexive, complete and transitive binary relation $\preceq$ on $2^{X}$ will be called an order on $2^{X}$. (Note: sometimes this is called a complete pre-order or a weak order.) Such 
an order gives rise to two other relations $\prec$ and $\sim$ on $2^{X}$, defined for all $x, y \in X$ by

(a) $x \prec y \Longleftrightarrow x \preceq y$ and not $(y \preceq x)$;

(b) $x \sim y \Longleftrightarrow x \preceq y$ and $y \preceq x$.

If an order $\preceq$ is anti-symmetric, then all equivalence classes (under the relation $\sim$ ) are singletons, and $\preceq$ is called a linear order.

Definition 2. Let $X$ be a finite set. A linear order $\preceq$ on $2^{X}$ is called a comparative probability order if $\emptyset \preceq A$ for every non-empty subset $A$ of $X$, and $\preceq$ satisfies de Finetti's axiom, namely

$$
A \preceq B \Longleftrightarrow A \cup C \preceq B \cup C,
$$

for all $A, B, C \in 2^{X}$ such that $(A \cup B) \cap C=\emptyset$.

For convenience, we will further suppose that $X=[n]=\{1,2, \ldots, n\}$ and denote the set of all comparative probability orders on $2^{[n]}$ as $\mathcal{P}_{n}$.

If we have a probability measure $\mathbf{p}=\left(p_{1}, \ldots, p_{n}\right)$ on $X$, where $p_{i}$ is the probability of $i$, then we know the probability of every event $A$, by the rule $p(A)=\sum_{i \in A} p_{i}$. We may now define an order $\preceq_{\mathbf{p}}$ on $2^{X}$ by

$$
A \preceq_{\mathbf{p}} B \quad \text { if and only if } \quad p(A) \leq p(B) .
$$

If $p_{i}>0$ for all $i$, and the probabilities of all events are different, then $\preceq_{\mathbf{p}}$ is a comparative probability order on $X$. Any such order is called (additively) representable. The set of representable comparative probability orders is denoted $\mathcal{L}_{n}$. It is known [10] that $\mathcal{L}_{n}$ is strictly contained in $\mathcal{P}_{n}$ for all $n \geq 5$.

We will always assume here that a linear order $\preceq$ on $2^{X}$ is a comparative probability order. As in [7] 8, it is often convenient to assume without loss of generality that

$$
1 \prec 2 \prec \ldots \prec n,
$$

which is equivalent to assuming that $p_{1}<p_{2}<\ldots<p_{n}$ when $\preceq$ is the comparative probability order represented by the probability measure $\mathbf{p}=\left(p_{1}, \ldots, p_{n}\right)$. The set of all comparative probability orders on $[n]$ that satisfy (3), will be denoted $\mathcal{P}_{n}^{*}$. The set of all representable comparative probability orders on $[n]$ that satisfy (3), will be denoted $\mathcal{L}_{n}^{*}$.

To every linear order $\preceq \in \mathcal{P}_{n}^{*}$, there corresponds a discrete cone $\mathcal{C}(\preceq)$ in $T^{n}$, where $T=\{-1,0,1\}$ (as defined in [7]).

Definition 3. A subset $\mathcal{C} \subseteq T^{n}$ is said to be a discrete cone if the following properties hold:

D1. $\left\{\mathbf{e}_{1}, \mathbf{e}_{2}-\mathbf{e}_{1}, \ldots, \mathbf{e}_{n}-\mathbf{e}_{n-1}\right\} \subseteq \mathcal{C}$, where $\left\{\mathbf{e}_{1}, \ldots, \mathbf{e}_{n}\right\}$ is the standard basis of $\mathbb{R}^{n}$,

D2. $\mathbf{0} \in \mathcal{C}$ and for every $\mathbf{0} \neq \mathbf{x} \in T^{n}$, either $\mathbf{x}$ or $-\mathbf{x}$ (but not both) is in $\mathcal{C}$,

D3. $\mathbf{x}+\mathbf{y} \in \mathcal{C}$ whenever $\mathbf{x}, \mathbf{y} \in \mathcal{C}$ and $\mathbf{x}+\mathbf{y} \in T^{n}$.

We note that Fishburn requires $\mathbf{0} \notin \mathcal{C}$ in $[7$ because his orders are anti-reflexive. In our case, condition D2 implies $\mathbf{0} \in \mathcal{C}$. 
For each subset $A \subseteq X$ we define the characteristic vector $\chi_{A}$ of this subset by

$$
\chi_{A}(i)= \begin{cases}1 & i \in A, \\ 0 & i \notin A,\end{cases}
$$

for $i=1,2, \ldots, n$. Given a comparative probability order $\preceq$ on $X$, we may define a characteristic vector $\chi(A, B)=\chi_{B}-\chi_{A} \in T^{n}$ for every pair of subsets $A, B$ for which $A \preceq B$ is true. The set of all characteristic vectors $\chi(A, B)$, for $A, B \in 2^{X}$ such that $A \preceq B$, is denoted by $\mathcal{C}(\preceq)$. The two axioms of comparative probability guarantee that $\mathcal{C}(\preceq)$ is a discrete cone. Given a discrete cone $\mathcal{C}$ we can also reconstruct a comparative probability order $\preceq$ for which $\mathcal{C}=\mathcal{C}(\preceq)$; see [7. Lemma 2.1].

Definition 4. A comparative probability order $\preceq$ satisfies the $m$ th cancellation condition $C_{m}$ if and only if there is no set $\left\{\mathbf{x}_{1}, \ldots, \mathbf{x}_{m}\right\}$ of non-zero vectors in $\mathcal{C}(\preceq)$ for which there exist positive integers $a_{1}, \ldots, a_{m}$ such that

$$
a_{1} \mathbf{x}_{1}+a_{2} \mathbf{x}_{2}+\cdots+a_{m} \mathbf{x}_{m}=0 .
$$

It is known (as shown in [10, and see also [4, 13, 7]) that a comparative probability order $\preceq$ is representable if and only if all cancellation conditions for $\mathcal{C}(\preceq)$ are satisfied.

Suppose that $\preceq \in \mathcal{P}_{n}$ is a not necessarily representable comparative probability order with the corresponding discrete cone $\mathcal{C}=\mathcal{C}(\preceq)$. Then the only way we can deduce one comparison from several others is by means of transitivity. Use of transitivity corresponds to the addition of the corresponding characteristic vectors of the cone. Indeed, suppose that $A \preceq B \preceq C$. Then $\chi(A, B)+\chi(B, C)=\chi(A, C)$. It might seem that closedness of $\mathcal{C}=\mathcal{C}(\preceq)$ under the addition is stronger than transitivity of $\preceq$, but in the presence of the de Finetti axiom, these two conditions are equivalent (see, for example, [7]).

Let us define a restricted sum for vectors in a discrete cone $\mathcal{C}$. Let $\mathbf{u}, \mathbf{v} \in \mathcal{C}$. Then

$$
\mathbf{u} \oplus \mathbf{v}=\left\{\begin{array}{cc}
\mathbf{u}+\mathbf{v} & \text { if } \mathbf{u}+\mathbf{v} \in T^{n}, \\
\text { undefined } & \text { if } \mathbf{u}+\mathbf{v} \notin T^{n} .
\end{array}\right.
$$

Definition 5. We say that the cone $\mathcal{C}$ is weakly generated by vectors $\mathbf{v}_{1}, \ldots, \mathbf{v}_{k}$ if every non-zero vector $\mathbf{c} \in \mathcal{C}$ can be expressed as a restricted sum of $\mathbf{v}_{1}, \ldots, \mathbf{v}_{k}$, in which each generating vector can be used as many times as needed. We denote this by $\mathcal{C}=\left\langle\mathbf{v}_{1}, \ldots, \mathbf{v}_{k}\right\rangle_{w}$.

If $\preceq$ is known to be representable, then there is an additional way to obtain new vectors in $\mathcal{C}(\preceq)$ from the existing ones, using the cancellation conditions. This can be formulated in terms of multilists of comparisons (see, for example, 9] proof of Theorem 3.7]). In terms of cones, this tool is given in Lemma 1] which says that in representable cones we can deduce new comparisons by forming linear combinations of the characteristic vectors of known comparisons with positive coefficients. This is also a reformulation of Axiom 3 from [9] in terms of discrete cones associated with $\preceq$.

Lemma 1. Let $\preceq \in \mathcal{L}_{n}$ be a representable comparative probability order, and $\mathcal{C}(\preceq)$ the corresponding discrete cone. Suppose $\left\{\mathbf{x}_{1}, \ldots, \mathbf{x}_{m}\right\} \subseteq \mathcal{C}(\preceq)$ and suppose that $\mathbf{x}=a_{1} \mathbf{x}_{1}+a_{2} \mathbf{x}_{2}+\cdots+a_{m} \mathbf{x}_{m}$ for some positive real numbers $a_{1}, \ldots, a_{m}$ and $\mathbf{x} \in T^{n}$. Then $\mathbf{x} \in \mathcal{C}(\preceq)$.

Proof. Suppose that the positive real numbers $a_{1}, \ldots, a_{m}$ satisfy $\mathbf{x}=a_{1} \mathbf{x}_{1}+a_{2} \mathbf{x}_{2}+\cdots+a_{m} \mathbf{x}_{m}$. Then these will be solutions of a system of linear equations with rational (even integer) coefficients. Hence there will be rational numbers satisfying the same equation, and therefore we may assume that 
$a_{1}, \ldots, a_{m}$ are rational. Let $a_{i}=p_{i} / q_{i}$, where $p_{i}$ and $q_{i}$ are positive integers. Then multiplying by the least common multiple of all the denominators, we see that the given equation can be written as $s_{0} \mathbf{x}=s_{1} \mathbf{x}_{1}+s_{2} \mathbf{x}_{2}+\cdots+s_{m} \mathbf{x}_{m}$, where $s_{0}, s_{1}, \ldots, s_{m}$ are integers. If $\mathbf{x} \notin \mathcal{C}(\preceq)$, then $-\mathbf{x} \in \mathcal{C}(\preceq)$ and so this equation can be written as $s_{0}(-\mathbf{x})+s_{1} \mathbf{x}_{1}+s_{2} \mathbf{x}_{2}+\cdots+s_{m} \mathbf{x}_{m}=\mathbf{0}$, which contradicts the $(m+1)$ th cancellation condition. Thus $\mathbf{x} \in \mathcal{C}(\preceq)$.

Definition 6. We say that the cone $\mathcal{C}$ is strongly generated by vectors $\mathbf{v}_{1}, \ldots, \mathbf{v}_{k}$ if every non-zero vector $\mathbf{c} \in \mathcal{C}$ can be obtained from $\mathbf{v}_{1}, \ldots, \mathbf{v}_{k}$ using the operation of restricted sum and linear combinations with positive rational coefficients. We denote this by $\mathcal{C}=\left\langle\mathbf{v}_{1}, \ldots, \mathbf{v}_{k}\right\rangle$.

In the process of elicitation of an individual's comparative probability order $\preceq$, a single query will be of the form "Is $A \prec B$ ?" for distinct non-empty subsets $A, B \in 2^{[n]}$. Hence we will sometimes assume the comparison $A \prec B$ means a positive answer to this query. In particular, for a representable comparative probability order $\preceq$, we will say that the comparisons $A_{1} \prec B_{1}, \ldots, A_{\ell} \prec B_{\ell}$ determine $\preceq$ in $\mathcal{L}_{n}$ if the vectors $\chi\left(A_{1}, B_{1}\right), \ldots, \chi\left(A_{\ell}, B_{\ell}\right)$ strongly generate $\mathcal{C}(\preceq)$.

Example 1. Let us consider the order

$$
\emptyset \prec 1 \prec 2 \prec 12 \prec 3 \prec 13 \prec 23 \prec 123 .
$$

and its respective cone $\mathcal{C}=\mathcal{C}(\preceq)$. Let us choose $\mathbf{v}_{1}=(1,0,0), \mathbf{v}_{2}=(-1,1,0), \mathbf{v}_{3}=(-1,-1,1)$, which correspond to comparisons

$$
\emptyset \prec 1, \quad 1 \prec 2, \quad 12 \prec 3,
$$

respectively. Then all other non-zero vectors of $\mathcal{C}$ can be expressed using $\mathbf{v}_{1}, \mathbf{v}_{2}, \mathbf{v}_{3}$. For example,

$$
\begin{aligned}
\mathbf{v}_{1} \oplus \mathbf{v}_{2} & =(0,1,0), \\
\mathbf{v}_{1} \oplus \mathbf{v}_{3} & =(0,-1,1), \\
\mathbf{v}_{2} \oplus \mathbf{v}_{3} & =(-1,0,1), \\
\mathbf{v}_{1} \oplus\left(\mathbf{v}_{1} \oplus \mathbf{v}_{2}\right) & =(1,1,0), \\
\left(\mathbf{v}_{1} \oplus\left(\mathbf{v}_{1} \oplus \mathbf{v}_{2}\right)\right) \oplus \mathbf{v}_{3} & =(0,0,1),
\end{aligned}
$$

and so on. Thus $\mathcal{C}=\left\langle\mathbf{v}_{1}, \mathbf{v}_{2}, \mathbf{v}_{3}\right\rangle_{w}$. The order $\preceq$ is representable, but we did not use linear combinations to generate $\mathcal{C}$. Therefore the comparisons (5) determine $\preceq$ not only in the class of representable comparative probability orders $\mathcal{L}_{n}$, but also in the class of all comparative probability orders $\mathcal{P}_{n}$.

Let us now give an example which shows that for representable cones, weak and strong generation are different. We will construct a representable cone whose minimal set of weak generators cannot be a minimal set of strong generators.

Example 2. In an example constructed by Kraft, Pratt and Seidenberg ([10. p. 415]), we re-label $q=1$, $r=2, s=3, p=4$ and $t=5$, to obtain a non-representable comparative probability order $\preceq$ on $[5]$ :

$$
\emptyset \prec 1 \prec 2 \prec 3 \prec 12 \prec 13 \prec 4 \prec 14 \prec 23 \prec 5 \prec 123 \prec 24 \prec 34 \prec 15 \prec 124 \prec 25 \prec 134 \ldots
$$

(Here only the first 17 terms are shown, since the remaining ones can be uniquely reconstructed). This order does not satisfy the 4 th cancellation condition, since it contains the following comparisons:

$$
13 \prec 4, \quad 14 \prec 23, \quad 34 \prec 15 \text { and } 25 \prec 134 \text {, }
$$


whose characteristic vectors $\mathbf{u}_{1}=(-1,0,-1,1,0), \mathbf{u}_{2}=(-1,1,1,-1,0), \mathbf{u}_{3}=(1,0,-1,-1,1)$ and $\mathbf{u}_{4}=$ $(1,-1,1,1,-1)$ satisfy

$$
\mathbf{u}_{1}+\mathbf{u}_{2}+\mathbf{u}_{3}+\mathbf{u}_{4}=\mathbf{0}
$$

The structure of the corresponding cone $\mathcal{C}=\mathcal{C}(\preceq)$ is as follows: $\mathcal{C}$ includes all vectors of $T^{n}$ lying in the half-space $S_{\mathbf{b}}=\left\{\mathbf{x} \in \mathbb{R}^{n} \mid(\mathbf{x}, \mathbf{b})>0\right\}$, and the four vectors $\mathbf{u}_{1}, \mathbf{u}_{2}, \mathbf{u}_{3}, \mathbf{u}_{4}$ lying on the hyperplane $H_{\mathbf{b}}=\left\{\mathbf{x} \in \mathbb{R}^{n} \mid(\mathbf{x}, \mathbf{b})=0\right\}$ with normal vector

$$
\mathbf{b}=\frac{1}{16}(1,2,3,4,6)
$$

In this linear order, 25 is the 16th subset and 134 is the 17 th, so $25 \prec 134$ is the central comparison. Obviously we can 'flip' this central comparison, replacing $25 \prec 134$ by $134 \prec 25$, and still have a comparative probability order $\preceq^{\prime}$, with cone $\mathcal{C}^{\prime}=\left(\mathcal{C} \backslash\left\{\mathbf{u}_{4}\right\}\right) \cup\left\{-\mathbf{u}_{4}\right\}$. Moreover, this will be a representable comparative probability order, since all cancellation conditions will be satisfied. Indeed, if we had a set of vectors $\left\{\mathbf{v}_{1}, \ldots, \mathbf{v}_{k}\right\} \subset \mathcal{C}^{\prime}$ such that

$$
\mathbf{v}_{1}+\mathbf{v}_{2}+\ldots+\mathbf{v}_{k}=\mathbf{0}
$$

then we would have $\left\{\mathbf{v}_{1}, \ldots, \mathbf{v}_{k}\right\} \subset H_{\mathbf{b}}$ and therefore $\left\{\mathbf{v}_{1}, \ldots, \mathbf{v}_{k}\right\} \subseteq\left\{\mathbf{u}_{1}, \mathbf{u}_{2}, \mathbf{u}_{3},-\mathbf{u}_{4}\right\}$, which is impossible.

Since $\mathbf{u}_{1}+\mathbf{u}_{2} \notin T^{n}, \mathbf{u}_{1}+\mathbf{u}_{3} \notin T^{n}$ and $\mathbf{u}_{2}+\mathbf{u}_{3} \notin T^{n}$, any set of weak generators of $\mathcal{C}^{\prime}$ must include all of $\mathbf{u}_{1}, \mathbf{u}_{2}, \mathbf{u}_{3},-\mathbf{u}_{4}$. But

$$
-\mathbf{u}_{4}=\mathbf{u}_{1}+\mathbf{u}_{2}+\mathbf{u}_{3}
$$

and hence any minimal set of weak generators of $\mathcal{C}$ cannot be a minimal set of strong generators since $-\mathbf{u}_{4}$ can be excluded from it.

Definition 7. Let $\mathcal{C}$ be a discrete cone. Define its weak rank rank $(\mathcal{C})$ to be the smallest number of vectors in $\mathcal{C}$ that weakly generate $\mathcal{C}$. Define its strong rank rank $(\mathcal{C})$ to be the smallest number of vectors in $\mathcal{C}$ that strongly generate $\mathcal{C}$.

Obviously $\operatorname{rank}_{s}(\mathcal{C}) \leq \operatorname{rank}_{w}(\mathcal{C})$, and the previous example shows that it is possible in some cases to

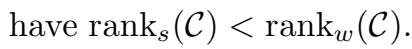

\section{Critical and flippable pairs}

Definition 8. Let $A$ and $B$ be disjoint subsets of $[n]$. The pair $(A, B)$ is said to be critica 3 for $\preceq$ if $A \prec B$ and there is no $C \subseteq[n]$ for which $A \prec C \prec B$.

Definition 9. Let $A$ and $B$ be disjoint subsets of $[n]$. The pair $(A, B)$ is said to be flippable for $\preceq$ if for every $D \subseteq[n]$ disjoint from $A \cup B$ the pair $(A \cup D, B \cup D)$ is critical.

Since in the latter definition we allow the possibility that $D=\emptyset$, every flippable pair is critical.

We note that the set of flippable pairs is not empty, since the central pair of any comparative probability order is flippable. Indeed, this consists of a certain set $A$ and its complement $A^{c}=X \backslash A$, and

\footnotetext{
${ }^{3}$ We follow Fishburn [9] in this definition, while Maclagan [1] calls such pairs primitive.
} 
there is no $D$ which has empty intersection with both of these sets. It is not known whether this can be the only flippable pair of the order.

Suppose now that a pair $(A, B)$ is flippable for a comparative probability order $\preceq$, and $A \neq \emptyset$. Then reversing each comparisons $A \cup D \prec B \cup D$ (to $B \cup D \prec A \cup D$ ), we will obtain a new comparative probability order $\preceq^{\prime}$, since the de Finetti axiom will still be satisfied. We say that $\preceq^{\prime}$ is obtained from $\preceq$ by flipping over $A \prec B$. The orders $\preceq$ and $\preceq^{\prime}$ are called flip-related. This flip relation turns $\mathcal{P}_{n}$ into a graph.

A pair $(A, B)$ with $A=\emptyset$ can be flippable with no possibility of flipping over. Below we mark with an asterisk the three flippable pairs of the comparative probability order of Example 1

$$
\emptyset \prec_{*} 1 \prec_{*} 2 \prec 12 \prec_{*} 3 \prec 13 \prec 23 \prec 123 .
$$

The first comparison $\emptyset \prec_{*} 1$ cannot be flipped over while the other two can be.

Definition 10. An element $\mathbf{w}$ of the cone $\mathcal{C}$ is said to be reducible if there exist two other vectors $\mathbf{u}, \mathbf{v} \in \mathcal{C}$ such that $\mathbf{w}=\mathbf{u} \oplus \mathbf{v}$, and irreducible otherwise.

Theorem 1. A pair $(A, B)$ of disjoint subsets is flippable for $\preceq$ if and only if the corresponding characteristic vector $\chi(A, B)$ is irreducible in $\mathcal{C}(\preceq)$.

Proof. Suppose $(A, B)$ is flippable but $\mathbf{w}=\chi(A, B)$ is reducible. Then $\mathbf{w}=\mathbf{u} \oplus \mathbf{v}$, where $\mathbf{u}=\chi(C, D)$ and $\mathbf{v}=\chi(E, F)$ for some $C, D, E, F$ such that $C \prec D$ and $E \prec F$. We may assume without loss of generality that $C \cap D=E \cap F=\emptyset$. Since $\mathbf{u}+\mathbf{v} \in \mathcal{C}(\preceq) \subset T^{n}$ and $C \cap D=E \cap F=\emptyset$, we have $C \cap E=D \cap F=\emptyset$. Also since $\chi(A, B)=\chi(C, D)+\chi(E, F)$, it is easy to see that

$$
A=(C \backslash F) \cup(E \backslash D) \text { and } B=(D \backslash E) \cup(F \backslash C) .
$$

Let $X=C \cap F$. Then $X \cap(A \cup B)=\emptyset$, and since $(C \cup D) \cap(E \backslash D)=(E \cup F) \cap(D \backslash E)=\emptyset$ we have

$$
A \cup X=C \cup(E \backslash D) \prec D \cup(E \backslash D)=(D \backslash E) \cup E \prec(D \backslash E) \cup F=B \cup X .
$$

In particular, $A \cup X$ and $B \cup X$ are not neighbors in $\preceq$, so $(A, B)$ is not flippable - contradiction.

Suppose now that $A \prec B$ but $(A, B)$ is not flippable. Then there exist subsets $C$ and $D$ such that $(A \cup B) \cap C=\emptyset$ and

$$
A \cup C \prec D \prec B \cup C .
$$

We may assume that $C$ is minimal with this property, and hence that $C \cap D=\emptyset$. Now if $\mathbf{u}=\chi(A \cup C, D)$ and $\mathbf{v}=\chi(D, B \cup C)$, then

$$
\mathbf{u} \oplus \mathbf{v}=\chi(A \cup C, B \cup C)=\chi(A, B)=\mathbf{w},
$$

and so $\mathbf{w}$ is reducible.

Theorem 2. Let $\preceq$ be a representable comparative probability order. Then the set of irreducible elements of $\mathcal{C}=\mathcal{C}(\preceq)$ is the smallest set that weakly generates $\mathcal{C}$. 
Proof. It is clear that the set of all irreducible elements $\operatorname{Irr}(\mathcal{C})$ of $\mathcal{C}=\mathcal{C}(\preceq)$ is contained in any set of weak generators. Let $\mathbf{x} \in \mathcal{C}$. We will prove that either $\mathbf{x}$ belongs to $\operatorname{Irr}(\mathcal{C})$ or $\mathbf{x}$ can be represented as a restricted sum of elements of $\operatorname{Irr}(\mathcal{C})$. Suppose $\mathbf{x} \notin \operatorname{Irr}(\mathcal{C})$. Then $\mathbf{x}=\mathbf{x}_{1} \oplus \mathbf{x}_{2}$ for some $\mathbf{x}_{i} \in \mathcal{C}$. If both of them belong to $\operatorname{Irr}(\mathcal{C})$, we are done. If at least one of them does not, then we continue representing both as restricted sums of vectors of $\mathcal{C}$. In this way, we obtain a binary tree of elements of $\mathcal{C}$. We claim that not a single branch of this tree can be longer than the cardinality of $\mathcal{C}$. If one of the branches were longer, then there would be two equal elements in it. Hence it would be possible to start a tree with some element and find the same element deep inside the tree. Without loss of generality, we can assume that $\mathbf{x}$ itself can be found in a tree generated by $\mathbf{x}$. If we stop when $\mathbf{x}$ has appeared for the second time, then we will have

$$
\mathbf{x}=G\left(\mathbf{x}, \mathbf{x}_{1}, \ldots, \mathbf{x}_{m}\right),
$$

where $G$ is some term in the algebra $\langle\mathcal{C}, \oplus\rangle$. Then if we express restricted addition through the ordinary one, the term $\mathbf{x}$ will cancel on both sides, and we will obtain an expression

$$
a_{1} \mathbf{x}_{1}+a_{2} \mathbf{x}_{2}+\ldots+a_{m} \mathbf{x}_{m}=\mathbf{0}
$$

with all coefficients $a_{i}$ positive integers. This will violate the $m$ th cancellation condition.

This theorem does not hold for non-representable orders as the following example shows.

Example 3. In the non-representable comparative probability order of Example Q we mark all flippable pairs with an asterisk:

$$
\emptyset \prec 1 \prec 2 \prec 3 \prec 12 \prec 13 \prec_{*} 4 \prec 14 \prec_{*} 23 \prec 5 \prec_{*} 123 \prec 24 \prec 34 \prec_{*} 15 \prec 124 \prec 25 \prec_{*} 134 \ldots
$$

There are five such pairs. Let $\mathbf{f}_{1}=\chi(13,4), \mathbf{f}_{2}=\chi(14,23), \mathbf{f}_{3}=\chi(5,123), \mathbf{f}_{4}=\chi(34,15), \mathbf{f}_{5}=\chi(25,134)$, and also let $\mathbf{x}=\chi(23,5)$. Then it is easy to check that

$$
\mathbf{x}=\mathbf{f}_{1} \oplus\left(\left(\mathbf{f}_{5} \oplus\left(\mathbf{f}_{2} \oplus \mathbf{x}\right)\right) \oplus \mathbf{f}_{4}\right),
$$

but on the other hand, $\mathbf{x}$ cannot be represented as a restricted sum of $\mathbf{f}_{1}, \ldots, \mathbf{f}_{5}$ since it is not in the subspace spanned by $\mathbf{f}_{1}, \ldots, \mathbf{f}_{5}$. The reason for (6) is of course the equation $\mathbf{f}_{1}+\mathbf{f}_{2}+\mathbf{f}_{4}+\mathbf{f}_{5}=\mathbf{0}$.

\section{Elicitation of comparative probability orders}

One of the problems in decision theory and the theory of measurement is to determine an individual's preference order over subsets of objects, or an individual comparative probability order over subsets of states of the world [9]. This stipulates the design of strategies to elicit those comparisons between subsets which determine the preference order completely.

Let $A, B \subseteq[n]$ be two non-empty disjoint subsets. Then given a comparative probability order $\preceq$, we can have both $A \prec B$ and $B \prec A$, so by asking an individual to compare $A$ and $B$ we obtain certain information about this individual's preference order. If, however, $A=\emptyset$ and $B$ is non-empty, then $A \prec B$ and such comparison gives no information about the order.

Definition 11. A set $S=\left\{A_{1} \prec B_{1}, \ldots, A_{\ell} \prec B_{\ell}\right\}$ of comparisons determines a comparative probability order $\preceq$ in $\mathcal{P}_{n}$ if the characteristic vectors $\chi\left(A_{i}, B_{i}\right)$ together with the standard basis of $\mathbb{R}^{n}$ weakly generate $\mathcal{C}(\preceq)$. 
Note that if such a set $S$ determines a comparative probability order, then one can deduce all other comparisons in $\preceq$ by virtue of transitivity alone (using the comparisons from $S$ and 'trivial' comparisons $\emptyset \prec\{i\})$. For representable orders we have the following:

Definition 12. A set $S=\left\{A_{1} \prec B_{1}, \ldots, A_{\ell} \prec B_{\ell}\right\}$ of comparisons determines a representable comparative probability order $\preceq$ in $\mathcal{L}_{n}$ if the characteristic vectors $\chi\left(A_{i}, B_{i}\right)$ together with the standard basis of $\mathbb{R}^{n}$ strongly generate $\mathcal{C}(\preceq)$.

Note that if such a set $S$ determines a representable comparative probability order, then one can deduce all other comparisons in $\preceq$ by means of positive linear combinations of the characteristic vectors $\chi\left(A_{i}, B_{i}\right)$ and the standard basis vectors.

Now let $S$ be a minimal set of comparisons that weakly (resp. strongly) determines a comparative probability order (resp. representable comparative probability order) $\preceq$. It is possible that all 'trivial' comparisons $\emptyset \prec\{i\}$ are implied by comparisons from $S$; in this case the corresponding minimal set of generators of the cone $\mathcal{C}(\preceq)$ has cardinality $|S|$. If not, then the corresponding minimal set of generators of $\mathcal{C}(\preceq)$ has $|S|+1$ generators. Indeed, both $\emptyset \prec\{i\}$ and $\emptyset \prec\{j\}$ cannot be in any minimal set of generators, since one of them implies the other.

Fishburn, Pekeč and Reeds [9] Theorem 3.7] proved that if $A_{1} \prec B_{1}, \ldots, A_{\ell} \prec B_{\ell}$ is a minimal set of subset comparisons that determines a representable comparative probability order $\preceq$ in $\mathcal{L}_{n}$, then the pairs $\left(A_{1}, B_{1}\right), \ldots,\left(A_{\ell}, B_{\ell}\right)$ are critical for $\preceq$. Now we can generalise this important theorem and also give a more transparent proof of it.

Theorem 3. Let $\preceq \in \mathcal{L}_{n}$ be a representable comparative probability order. Further, suppose that $\left\{A_{1} \prec B_{1}, \ldots, A_{\ell} \prec B_{\ell}\right\}$ is a minimal set of subset comparisons that determines $\preceq$ in $\mathcal{L}_{n}$. Then all of the pairs $\left(A_{1}, B_{1}\right), \ldots,\left(A_{\ell}, B_{\ell}\right)$ are flippable for $\preceq$.

Proof. A smallest set of subset comparisons corresponds to a minimal set of strong generators of the respective cone $\mathcal{C}=\mathcal{C}(\preceq)$, and this is either $G=\left\{\mathbf{g}_{1}, \ldots, \mathbf{g}_{\ell}\right\}$ or $G=\left\{\mathbf{g}_{1}, \ldots, \mathbf{g}_{\ell+1}\right\}$ with $\mathbf{g}_{\ell+1}=\mathbf{e}_{i}$ for some $i=1,2, \ldots, n$. Without loss of generality let us consider the first case. By Theorem 1 to show that a pair $\left(A_{1}, B_{1}\right)$ is flippable, it is enough to show that $\mathbf{g}_{1}=\mathbf{u}+\mathbf{v}$ for no two vectors $\mathbf{u}, \mathbf{v} \in \mathcal{C}$. Let us assume the contrary. Then $m \mathbf{u}=\sum_{i=1}^{\ell} m_{i} \mathbf{g}_{i}$ and $k \mathbf{v}=\sum_{i=1}^{\ell} k_{i} \mathbf{g}_{i}$ for some positive integers $m, m_{i}$ and $k, k_{j}$. Let $r=\operatorname{lcm}(m, k)$. Then multiplying the two equations by their respective factors, we obtain $r \mathbf{u}=\sum_{i=1}^{\ell} m_{i}^{\prime} \mathbf{g}_{i}$ and $r \mathbf{v}=\sum_{i=1}^{\ell} k_{i}^{\prime} \mathbf{g}_{i}$. Adding these we obtain

$$
r \mathbf{g}_{1}=\sum_{i=1}^{\ell}\left(m_{i}^{\prime}+k_{i}^{\prime}\right) \mathbf{g}_{i}
$$

We now consider two cases. If $r>m_{1}^{\prime}+k_{1}^{\prime}$, then $\mathbf{g}_{1}$ can be excluded from the set of generators $G$ which was supposed to be minimal, contradiction. If $r \leq m_{1}^{\prime}+k_{1}^{\prime}$, then the $\ell$ th cancellation condition is violated, again a contradiction. This proves the theorem. 


\section{Geometric representation of representable comparative probability orders}

Geometric representation of comparative probability orders was first introduced by Fine and Gill [5]. To explain it, we need to recall a few basic facts about hyperplane arrangements in $\mathbb{R}^{n}$; see 14 12 for more information about these. A hyperplane is a translate of an $(n-1)$-dimensional subspace of $\mathbb{R}^{n}$. Any hyperplane of the form $H=\left\{\mathbf{x} \in \mathbb{R}^{n} \mid(\mathbf{n}, \mathbf{x})=0\right\}$, where $\mathbf{n}$ is a non-zero vector, will be called linear, with the vector $\mathbf{n}$ called the normal vector of $H$. Any hyperplane of the form $J=\left\{\mathbf{x} \in \mathbb{R}^{n} \mid(\mathbf{n}, \mathbf{x})=a\right\}$ where $a \neq 0$ will be called an affine hyperplane. Every affine hyperplane is a translate of the linear hyperplane having the same normal vector $\mathbf{n}$.

A hyperplane arrangement $\mathcal{A}$ is any finite set of hyperplanes. A region of an arrangement $\mathcal{A}$ is a connected component of the complement of the union $U$ of the hyperplanes of $\mathcal{A}$ - that is, the open set

$$
U=\mathbb{R}^{n} \backslash \bigcup_{H \in \mathcal{A}} H
$$

In particular, every region of an arrangement is an open set. Each region has an associated polytope which is the closure of the region. This polytope is convex.

Let $\mathcal{A}$ be an arrangement of hyperplanes in $\mathbb{R}^{n}$, and let $J$ be any hyperplane in $\mathbb{R}^{n}$. Then the set

$$
\mathcal{A}^{J}=\{H \cap J \mid H \in \mathcal{A}\}
$$

is called the induced arrangement of hyperplanes in $J$.

Let $A, B \subseteq[n]$ be disjoint subsets, of which at least one is non-empty. Corresponding to the pair $(A, B)$ is a hyperplane $H(A, B)$ consisting of all points $\mathbf{x}$ in $\mathbb{R}^{n}$ satisfying the equation

$$
\sum_{a \in A} x_{a}-\sum_{b \in B} x_{b}=0
$$

Such hyperplanes have normal vectors in the set $\{-1,0,1\}^{n} \backslash \mathbf{0}$, where $\mathbf{0}$ is the $n$-dimensional zero vector. Let us denote the corresponding hyperplane arrangement by $\mathcal{A}_{n}$. Also let $J$ be the hyperplane in $\mathbb{R}^{n}$ defined by the equation $x_{1}+x_{2}+\ldots+x_{n}=1$, and let $\mathcal{H}_{n}=\mathcal{A}_{n}^{J}$ be the induced hyperplane arrangement. We are interested in the regions of $\mathcal{H}_{n}$ which lie in the positive orthant $\mathbb{R}_{+}^{n}$ of $\mathbb{R}^{n}$, given by $x_{i} \geq 0$ for $i=1,2, \ldots, n$. The closure of the union of all of these regions is an $(n-1)$-dimensional simplex $S^{n-1}$ in $J$.

As we know, every point $\mathbf{p}=\left(p_{1}, \ldots, p_{n}\right) \in S^{n-1}$ defines a representable comparative probability order $\preceq_{\mathbf{p}}$ from $\mathcal{L}_{n}$ given by the rule (2) from Section 2 If $\mathbf{p}$ and $\mathbf{q}$ are two points of $S^{n-1}$, then the orders $\preceq_{\mathbf{p}}$ and $\preceq_{\mathbf{q}}$ will coincide if and only if $\mathbf{p}$ and $\mathbf{q}$ are in the same region of the hyperplane arrangement $\mathcal{H}_{n}$. This immediately follows from the fact that the order $A \prec B$ changes to $B \prec A$ (or vice versa) precisely when we cross the hyperplane $H(A, B)$. Thus every comparative probability order in $\mathcal{L}_{n}$ is so represented by one of the regions of $\mathcal{H}_{n}$.

Let $P$ be the polytope representing $\preceq_{\mathbf{p}}$. A facet of the polytope will be called significant if it is not contained in any of the hyperplanes $x_{i}=0$. It is now clear that the minimal number of subset comparisons needed to define $\preceq_{\mathbf{p}}$ is the number of significant facets of the polytope representing $\preceq_{\mathbf{p}}$. We illustrate this using the following example. 
Example 4. The 12 regions of $\mathcal{H}_{3}$ on the figure below represent all 12 comparative probability orders on [3].

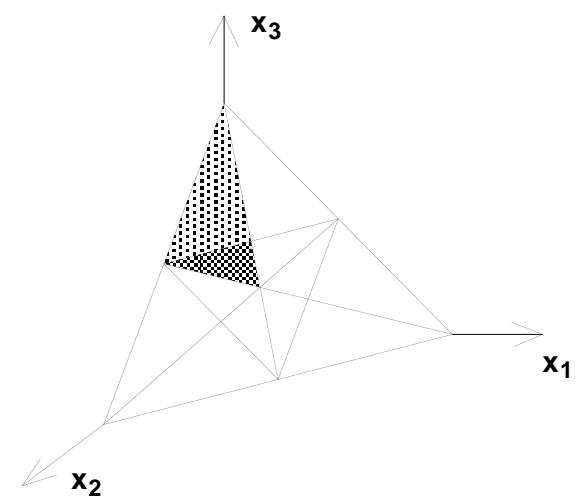

The two shaded triangular regions correspond to the two orders for which $1 \prec 2 \prec 3$, namely

$$
1 \prec 2 \prec 12 \prec 3 \prec 13 \prec 23 \prec 123 \quad \text { and } \quad 1 \prec 2 \prec 3 \prec 12 \prec 13 \prec 23 \prec 123 \text {, }
$$

with the lighter one corresponding to the first order (the lexicographic order). Note, however, that one of the boundaries for the first order is the hyperplane $x_{1}=0$, and it is determined by two comparisons, while the second order needs three comparisons, since all facets of the corresponding polytope are significant.

A simple but important statement is contained in the following

Lemma 2. Let $P$ be the polytope representing the order $\preceq_{\mathbf{p}}$, where $\mathbf{p}$ is a probability measure. Then $P$ can have at most one insignificant facet.

Proof. Suppose $P$ has at least one insignificant facet. We can show that if $p_{j}=\min _{i=1}^{n} p_{i}$, then one of its facets is contained in the hyperplane $x_{j}=0$, and this is the only insignificant facet of $P$. For suppose that another facet of $P$ is contained in $x_{k}=0$. Since $P$ lies in the half-space $x_{k}-x_{j} \geq 0$, we will have $x_{k}=x_{j}=0$ on this facet. It follows that this facet has $(n-2)$-dimensional volume zero, in contradiction to the fact that every facet of an $(n-1)$-dimensional polytope has non-zero $(n-2)$-dimensional volume.

The following theorem answers Open Problem 2 posed by Fishburn et al in [9, p. 243]:

Theorem 4. Let $\preceq$ be a representable comparative probability order. Then $\preceq$ is determined by no fewer than $n-1$ binary subset comparisons $A \prec B$, where $A, B$ are disjoint non-empty subsets of $[n]$. Moreover, if all facets of the polytope $P$ representing $\preceq$ are significant, then $\preceq$ is determined by no fewer than $n$ binary subset comparisons.

Proof. Consider the hyperplane arrangement $\mathcal{H}_{n}$ (as defined earlier in this section), and let $\mathbf{p}$ be a probability measure that corresponds to a representable order $\preceq \in \mathcal{L}_{n}$. The closure of the region $P$ to which $\mathbf{p}$ belongs is an convex polytope. Since it is open, it has a non-zero $(n-1)$-dimensional volume in $J$ and, therefore must have at least $n$ vertices and, hence, at least $n$ facets.

If all facets are significant, then each facet corresponds to a non-trivial subset comparison, hence we need at least $n$ subset comparisons to determine $\preceq$. Otherwise, by Lemma 2 there is a single insignificant facet, and in that case $n-1$ subset comparisons are needed to determine $\preceq$. 
For orders in $\mathcal{L}_{n}^{*}$ — that is, for those satisfying condition (3) from Section 2 — we have the following:

Corollary 1. Let $\preceq$ be a representable comparative probability order in $\mathcal{L}_{n}^{*}$ which is determined by exactly $n-1$ binary subset comparisons. Then the hyperplane $x_{1}=0$ contains one of the facets of the polytope corresponding to $\preceq$.

This can be expressed in terms of discrete cones as follows:

Corollary 2. Let $\preceq$ be a representable comparative probability order in $\mathcal{L}_{n}^{*}$ which is determined by exactly $n-1$ binary subset comparisons. Then the vector $\mathbf{g}_{1}=(1,0, \ldots 0)$ is present in any set of generators of the corresponding discrete cone $\mathcal{C}(\preceq)$.

\section{The product of two comparative probability orders}

We will use a construction for the product of two orders. An outline of this construction was given by Maclagan in [11, p. 294]. We now give a rigorous definition.

Definition 13. Suppose we have two comparative probability orders $\preceq_{1} \in \mathcal{P}_{k}$ and $\preceq_{2} \in \mathcal{P}_{m}$. Let us define a new order $\preceq=\preceq_{1} \times \preceq_{2}$ on $[k+m]$. First we transfer the order $\preceq_{2}$ from the set $[m]$ to the set $[k+m] \backslash[k]$ as follows: for any set $A=\left\{i_{1}, \ldots, i_{s}\right\} \subseteq[k+m] \backslash[k]$ we define its 'shift' $\bar{A}=\left\{i_{1}-k, \ldots, i_{s}-k\right\} \subseteq[m]$, and then for any two subsets $A, B \subseteq[k+m] \backslash[k]$, we define $A \preceq_{2} B$ if and only if $\bar{A} \preceq_{2} \bar{B}$. (NB: there will be no confusion in using the same notation for both the original order and the shifted order here.) Now let $A, B \subseteq[k+m]$. Such sets can be uniquely represented as $A=A_{1} \cup A_{2}$ and $B=B_{1} \cup B_{2}$, where $A_{1}, B_{1} \subseteq[k]$ and $A_{2}, B_{2} \subseteq[k+m] \backslash[k]$. Then finally we let $A \prec B$ if and only if either $A_{2} \prec_{2} B_{2}$, or $A_{2}=B_{2}$ and $A_{1} \prec_{1} B_{1}$. We call $\preceq$ the product of $\preceq_{1}$ and $\preceq_{2}$.

Proposition 1. Let $\preceq_{1} \in \mathcal{P}_{k}$ and $\preceq_{2} \in \mathcal{P}_{m}$ be two comparative probability orders with cones $\mathcal{C}_{1} \subset T^{k}$ and $\mathcal{C}_{2} \subset T^{m}$, respectively. Then their product $\preceq=\preceq_{1} \times \preceq_{2}$ is also a comparative probability order whose cone $\mathcal{C}(\preceq)$ consists of all vectors $(\mathbf{g}, \mathbf{0})$ with $\mathbf{g} \in \mathcal{C}_{1}$ and all vectors $(\mathbf{g}, \mathbf{h})$ with $\mathbf{g} \in T^{k}$ and $\mathbf{0} \neq \mathbf{h} \in \mathcal{C}_{2}$.

Proof. Let us consider the set of all characteristic vectors $\chi(A, B)$, where $A \preceq B$. These sets can be uniquely represented as $A=A_{1} \cup A_{2}$ and $B=B_{1} \cup B_{2}$, where $A_{1}, B_{1} \in[k]$ and $A_{2}, B_{2} \in[k+m] \backslash[k]$. If $A_{2} \prec_{2} B_{2}$, then $\chi(A, B)=(\mathbf{g}, \mathbf{h})$, where $\mathbf{g} \in T^{k}$ and $\mathbf{0} \neq \mathbf{h} \in \mathcal{C}_{2}$. If $A_{2}=B_{2}$, then $\chi(A, B)=(\mathbf{g}, \mathbf{0})$, where $\mathbf{g} \in \mathcal{C}_{1}$. We claim that the set $\mathcal{C}$ of all $\chi(A, B)$ so obtained is a discrete cone. Indeed, first the property $\mathrm{D} 1$ for $\mathcal{C}$ follows from the same property for $\mathcal{C}_{1}$ and $\mathcal{C}_{2}$. Second, if $\mathbf{v}=(\mathbf{x}, \mathbf{0})$ with $\mathbf{x} \in T^{k}$, then either $\mathbf{v}$ or $-\mathbf{v}$ belongs to $\mathcal{C}$, since either $\mathbf{x}$ or $-\mathbf{x}$ belongs to $\mathcal{C}_{1}$ (by property D2). On the other hand, if $\mathbf{v}=(\mathbf{g}, \mathbf{h})$ is any vector from $T^{k+m}$ for which $\mathbf{0} \neq \mathbf{h} \in T^{m}$, then if $\mathbf{h} \in \mathcal{C}_{2}$ then clearly $\mathbf{v}$ is in $\mathcal{C}$, while if $\mathbf{h} \notin \mathcal{C}_{2}$ then $-\mathbf{h} \in \mathcal{C}_{2}$ and $-\mathbf{v}=(-\mathbf{g},-\mathbf{h})$ is in $\mathcal{C}$. Hence D2 holds for $\mathcal{C}$. We leave the proof of D3 to the reader. Since $\mathcal{C}$ is a discrete cone, $\preceq$ is a comparative probability order, and $\mathcal{C}=\mathcal{C}(\preceq)$.

Definition 14. The order $\preceq \in \mathcal{L}_{n}$ is called reducible if it can be represented as a product of two other orders.

Example 5. The lexicographic order $\preceq \in \mathcal{L}_{n}$

$$
1 \prec 2 \prec 12 \prec 3 \prec 13 \prec 23 \prec 23 \prec 4 \prec \ldots
$$


can be represented as the product

$$
\left.\left(\ldots\left(\preceq_{0} \times \preceq_{0}\right) \times \preceq_{0}\right) \times \ldots\right) \times \preceq_{0}
$$

of $n$ copies of $\preceq_{0}$, where $\preceq_{0}$ is the only order in $\mathcal{L}_{1}$ (namely: $\emptyset \prec_{0}\{1\}$ ).

Theorem 5. Let $\preceq_{1} \in \mathcal{P}_{k}$ and $\preceq_{2} \in \mathcal{P}_{m}$ be two comparative probability orders. Then the weak rank (resp. strong rank) of $\preceq_{1} \times \preceq_{2}$ is the sum of the weak ranks (resp. strong ranks) of $\preceq_{1}$ and $\preceq_{2}$.

Proof. We will prove the theorem for the case of a weak rank. The proof for the case of a strong rank is similar.

Let $\mathcal{C}_{1}, \mathcal{C}_{2}, \mathcal{C}$ be the discrete cones of $\preceq_{1}, \preceq_{2}$ and $\preceq_{1} \times \preceq_{2}$, respectively. Let $\left\{\mathbf{g}_{1}, \ldots, \mathbf{g}_{s}\right\}$ and $\left\{\mathbf{h}_{1}, \ldots, \mathbf{h}_{t}\right\}$ be minimal sets of weak generators for $\mathcal{C}_{1}$ and $\mathcal{C}_{2}$. Let $G$ be the $s \times k$ matrix whose rows are the generators $\left\{\mathbf{g}_{1}, \ldots, \mathbf{g}_{s}\right\}$, and let $H$ be the $t \times m$ matrix whose rows are the generators $\left\{\mathbf{h}_{1}, \ldots, \mathbf{h}_{t}\right\}$. Also let $1_{t \times k}$ be the $t \times k$ matrix whose all entries are 1 , and let $0_{s \times m}$ be an $s \times m$ zero matrix. Now we are going to check that the rows of the $(s+t) \times(k+m)$ matrix

$$
M=\left[\begin{array}{cc}
G & 0_{s \times m} \\
-1_{t \times k} & H
\end{array}\right]
$$

generate $\mathcal{C}$.

It is obvious that any row $(\mathbf{g}, \mathbf{0})$, where $\mathbf{0} \neq \mathbf{g} \in \mathcal{C}_{1}$, can be obtained from the first $s$ rows using the operation $\oplus$. In particular, the row $(\mathbf{1}, \mathbf{0})$ can be so obtained, where $\mathbf{1}$ is the $k$-dimensional vector whose entries are all 1 . We will now show that every vector $(-\mathbf{1}, \mathbf{h})$, where $\mathbf{0} \neq \mathbf{h} \in \mathcal{C}_{2}$ can be generated. For this, all we need do is show is that if $\left(-\mathbf{1}, \mathbf{h}_{1}\right)$ and $\left(-\mathbf{1}, \mathbf{h}_{2}\right)$ can be generated, and $\mathbf{h}_{1} \oplus \mathbf{h}_{2}$ is defined, then also $\left(-\mathbf{1}, \mathbf{h}_{1} \oplus \mathbf{h}_{2}\right)$ can be generated; but that is easy, since

$$
\left(-\mathbf{1}, \mathbf{h}_{1} \oplus \mathbf{h}_{2}\right)=\left((\mathbf{1}, \mathbf{0}) \oplus\left(-\mathbf{1}, \mathbf{h}_{1}\right)\right) \oplus\left(-\mathbf{1}, \mathbf{h}_{2}\right)
$$

Suppose now that $\mathbf{c}=(\mathbf{g}, \mathbf{h}) \in T^{k+m}$, where $\mathbf{g} \in T^{k}$ and $\mathbf{0} \neq \mathbf{h} \in \mathcal{C}_{2}$. We wish to show that $\mathbf{c}$ can be generated using the rows of $M$ as generators. It is not difficult to see that there exist vectors $\mathbf{g}_{1}, \mathbf{g}_{2} \in \mathcal{C}_{1}$ such that

$$
\mathbf{g}=\left(-\mathbf{1} \oplus \mathbf{g}_{1}\right) \oplus \mathbf{g}_{2}
$$

If the $i$ th coordinate of $\mathbf{g}$ is 1 , then both $\mathbf{g}_{1}$ and $\mathbf{g}_{2}$ should have a 1 in the same position. If the $i$ th coordinate of $\mathbf{g}$ is 0 , then one of the vectors $\mathbf{g}_{1}$ or $\mathbf{g}_{2}$ should have a 1 in that position. Finally, If the $i$ th coordinate of $\mathbf{g}$ is -1 , then both $\mathbf{g}_{1}$ and $\mathbf{g}_{2}$ should have a 0 in that position. Since all coordinates of $\mathbf{g}_{1}$ and $\mathbf{g}_{2}$ are positive, they belong to $\mathcal{C}_{1}$. The vector $\mathbf{c}$ can now be obtained as follows:

$$
\mathbf{c}=(\mathbf{g}, \mathbf{h})=\left((-\mathbf{1}, \mathbf{h}) \oplus\left(\mathbf{g}_{1}, \mathbf{0}\right)\right) \oplus\left(\mathbf{g}_{2}, \mathbf{0}\right)
$$

This completes the proof. Note that it is also clear that the given set of generators (the rows of $M$ ) is minimal.

Example 6. Let us consider the order

$$
\emptyset \prec 1 \prec 2 \prec 3 \prec 12 \prec 13 \prec 23 \prec 123 .
$$


and its respective cone $\mathcal{C}=\mathcal{C}(\preceq)$. This cone has the following set of generators (both weak and strong):

$$
\begin{aligned}
& \mathbf{h}_{1}=(-1,1,0), \\
& \mathbf{h}_{2}=(0,-1,1), \\
& \mathbf{h}_{3}=(1,1,-1) .
\end{aligned}
$$

Let $\preceq_{0}$ be the order $\emptyset \prec_{0} 1$ of $\mathcal{L}_{1}$. Then as in the proof of Theorem 5 , the rows $\mathbf{u}_{1}, \ldots, \mathbf{u}_{4}$ of the matrix

$$
M=\left[\begin{array}{rrrr}
1 & 0 & 0 & 0 \\
-1 & -1 & 1 & 0 \\
-1 & 0 & -1 & 1 \\
-1 & 1 & 1 & -1
\end{array}\right]
$$

generate the cone for the order $\preceq_{0} \times \preceq$.

Another important property of the product is that it preserves representability.

Theorem 6. The product of two representable comparative probability orders $\preceq_{1} \in \mathcal{L}_{k}$ and $\preceq_{2} \in \mathcal{L}_{m}$ is a representable comparative probability order in $\mathcal{L}_{k+m}$.

Proof. Let $\mathbf{p}=\left(p_{1}, \ldots, p_{k}\right)$ and $\mathbf{q}=\left(q_{1}, \ldots, q_{m}\right)$ be the probability measures that represent $\preceq_{1}$ and $\preceq_{2}$, respectively. Since $\mathbf{q}$ is linear, the following number is non-zero:

$$
\varepsilon=\min _{I, J}\left\{\left|\sum_{i \in I} q_{i}-\sum_{j \in J} q_{j}\right|: I, J \subseteq[m], I \cap J=\emptyset\right\} .
$$

It is then easy to check that the measure given by

$$
\frac{1}{\varepsilon+1}\left(\varepsilon p_{1}, \varepsilon p_{2}, \ldots, \varepsilon p_{k}, q_{1}, q_{2}, \ldots, q_{m}\right)
$$

defines the order $\preceq_{1} \times \preceq_{2}$.

\section{Structural characterisation of comparative probability orders determined by $n-1$ binary comparisons}

We can now give a structural characterisation of the comparative probability orders in $\mathcal{L}_{n}^{*}$ whose corresponding polytope has a facet contained in the hyperplane $x_{1}=0$.

Theorem 7. Let $\preceq$ be a representable comparative probability order in $\mathcal{L}_{n}^{*}$ whose corresponding polytope $P$ has a facet contained in the hyperplane $x_{1}=0$. Then $\preceq=\preceq_{0} \times \preceq^{\prime}$, where $\preceq_{0}$ is the only order in $\mathcal{L}_{1}$ and $\preceq^{\prime}$ is a comparative probability order in $\mathcal{L}_{n-1}^{*}$.

Proof. Let $S$ be the minimal set of comparisons that define $\preceq$. By Theorem 3 all comparisons in $S$ are critical. Let $A \prec B$ be any one comparison from $S$, where $A, B \subseteq[n]$ are disjoint and non-empty, and consider the hyperplane associated with this comparison, consisting of all points $\mathbf{x} \in \mathbb{R}^{n}$ for which

$$
\sum_{a \in A} x_{a}=\sum_{b \in B} x_{b}
$$


Let $\mathbf{p}$ be any probability measure such that $\preceq=\preceq \mathbf{p}$. Since $A \prec B$, we see that $\mathbf{p}$ does not lie on the hyperplane (7), and that

$$
\sum_{a \in A} p_{a}<\sum_{b \in B} p_{b}
$$

We wish to show that also

$$
\sum_{a \in A \cup\{1\}} p_{a}<\sum_{b \in B \backslash\{1\}} p_{b} .
$$

First, assume that $1 \in B$. Since $A \prec B$ is critical, we have $B \backslash\{1\} \prec A$, and so

$$
\sum_{b \in B \backslash\{1\}} p_{b}<\sum_{a \in A} p_{a}<\sum_{b \in B} p_{b} .
$$

It follows that

$$
\sum_{b \in B \backslash\{1\}} x_{b}<\sum_{a \in A} x_{a}<\sum_{b \in B} x_{b}
$$

for every interior point $\mathbf{x}$ of this region, while

$$
\sum_{b \in B \backslash\{1\}} x_{b} \leq \sum_{a \in A} x_{a} \leq \sum_{b \in B} x_{b}
$$

for every point $\mathbf{x}$ on any facet of $P$. In particular, condition (10) must hold on the facet that is contained in the hyperplane $x_{1}=0$, which is a contradiction since no internal point of that facet is on the hyperplane $\sum_{b \in B \backslash\{1\}} x_{b}=\sum_{a \in A} x_{a}$. Hence $1 \notin B$.

Similarly, if we assume that $1 \notin A$, then the same kind of argument gives

$$
\sum_{a \in A} p_{a}<\sum_{b \in B} p_{b}<\sum_{a \in A \cup\{1\}} p_{a},
$$

again leading to a contradiction, so $1 \in A$.

Next, we consider $\mathcal{C}=\mathcal{C}(\preceq)$. We know that this cone has $n$ strong generators $\mathbf{g}_{1}, \ldots, \mathbf{g}_{n}$, with $\mathbf{g}_{1}=(1,0, \ldots 0)$. The other strong generators $\mathbf{g}_{2}, \ldots, \mathbf{g}_{n}$ correspond to characteristic vectors of critical pairs $A_{i} \prec B_{i}$ from $S$ with non-empty $A_{i}$ and $B_{i}$. Now we know that $1 \in A_{i}$ for $i=2, \ldots, n$, and hence the corresponding generators will be $\mathbf{g}_{i}=\left(-1, \mathbf{g}_{i}^{\prime}\right)$ for all $2 \leq i \leq n$, where $\mathbf{g}_{i}^{\prime} \in T^{n-1}$. Thus the rows of the following matrix are the strong generators of $\mathcal{C}$ :

$$
G=\left[\begin{array}{rr}
1 & 0 \\
-1 & \mathbf{g}_{2}^{\prime} \\
\vdots & \vdots \\
-1 & \mathbf{g}_{n}^{\prime}
\end{array}\right]
$$

Clearly $\mathbf{g}_{2}^{\prime}, \ldots \mathbf{g}_{n}^{\prime}$ generate a cone $\mathcal{C}^{\prime}=\mathcal{C}\left(\preceq^{\prime}\right)$ for some $\preceq^{\prime} \in \mathcal{L}_{n-1}$. As $\operatorname{rank}\left(\mathcal{C}^{\prime}\right) \leq n-1$, the order $\preceq^{\prime}$ is determined by no more than $n-1$ comparisons. The form of $G$ demonstrates that $\preceq=\preceq_{0} \times \preceq^{\prime}$.

Now we can give a characterisation of the comparative probability orders in $\mathcal{L}_{n}^{*}$ that can be determined by $n-1$ binary comparisons, answering Open Problem 3 posed by Fishburn et al in [9] p. 243]:

Theorem 8. Let $\preceq$ be a comparative probability order in $\mathcal{L}_{n}^{*}$ that can be determined by $n-1$ binary comparisons. Then $\preceq$ is reducible, and $\preceq=\preceq_{0} \times \preceq^{\prime}$, where $\preceq_{0}$ is the only order in $\mathcal{L}_{1}$ and $\preceq^{\prime}$ is 
a comparative probability order in $\mathcal{L}_{n-1}^{*}$ that can be determined by no more than $n-1$ comparisons. Conversely, if $\preceq^{\prime} \in \mathcal{L}_{n-1}^{*}$ is determined by no more than $n-1$ comparisons, then the product $\preceq=\preceq_{0} \times \preceq^{\prime}$ is a comparitive probability order in $\mathcal{L}_{n}^{*}$ that can be determined by $n-1$ binary comparisons, unless $\preceq^{\prime}$ requires exactly $n-1$ comparisons and is reducible as $\preceq^{\prime}=\preceq_{0} \times \preceq^{\prime \prime}$.

Proof. Let $\preceq \in \mathcal{L}_{n}^{*}$ be any order determined by a set of $n-1$ binary comparisons, and let $\mathbf{p}$ be a probability measure that determines $\preceq$, and $P$ the corresponding polytope in $J$. Then Corollary 1 implies that the hyperplane $x_{1}=0$ contains a facet of $P$. By Theorem $\square$ we know that $\preceq=\preceq_{0} \times \preceq^{\prime}$, where $\preceq_{0}$ is the only order in $\mathcal{L}_{1}$ and $\preceq^{\prime}$ is a comparative probability order in $\mathcal{L}_{n-1}^{*}$. Let $\mathcal{C}^{\prime}=\mathcal{C}\left(\preceq^{\prime}\right)$. Then $\mathcal{C}^{\prime}$ has rank $n-1$, and $\preceq^{\prime}$ is determined by $n-1$ or $n-2$ comparisons.

For the converse, if $\preceq^{\prime} \in \mathcal{L}_{n-1}$ is determined by at most $n-1$ comparisons, we have two cases to consider, as follows:

Case 1: $\preceq^{\prime}$ is determined by $n-1$ comparisons and $\preceq^{\prime} \neq \preceq_{0} \times \preceq^{\prime \prime}$.

In this case, if $H$ is a matrix with $n-1$ rows that are strong generators of $\mathcal{C}\left(\preceq^{\prime}\right)$, then

$$
G=\left[\begin{array}{rr}
1 & 0 \\
-1 & H
\end{array}\right]
$$

generates the cone $\mathcal{C}=\mathcal{C}\left(\preceq_{0} \times \preceq^{\prime}\right)$. Here the first row is $\mathbf{g}_{1}=(1,0, \ldots 0) \in \mathbb{R}^{n}$ and so $\preceq=\preceq_{0} \times \preceq^{\prime}$ is determined by $n-1$ binary comparisons. (We do not have to add a row $(1,0, \ldots, 0) \in \mathbb{R}^{n-1}$ to $H$ because the row $(-1,1,0, \ldots, 0) \in \mathbb{R}^{n}$ is strongly implied by the other rows of $G$.)

Case 2: $\preceq^{\prime}$ is determined by $n-2$ comparisons.

In this case, by Theorem $\mathbf{7}$ the cone $\mathcal{C}\left(\preceq^{\prime}\right)$ has $n-1$ strong generators $\mathbf{h}_{1}, \ldots, \mathbf{h}_{n-1}$ (with $\mathbf{h}_{1}=$ $\left.(1,0, \ldots, 0) \in \mathbb{R}^{n-1}\right)$, none of which can be removed. Hence the rows of

$$
G=\left[\begin{array}{rr}
1 & 0 \\
-1 & H
\end{array}\right]
$$

form a minimal set of strong generators of $\mathcal{C}$, and because the first row is $\mathbf{g}_{1}=(1,0, \ldots 0) \in \mathbb{R}^{n}$, the corresponding order $\preceq$ is determined by $n-1$ comparisons.

Example 7. We illustrate this proof using the two orders from Example 4. The first one (which is the lexicographic order), denoted say by $\prec_{1}$, is determined by the comparisons $1 \prec 2$ and $12 \prec 3$, and its cone is generated by the vectors

$$
\mathbf{v}_{1}=(1,0,0), \quad \mathbf{v}_{2}=(-1,1,0), \quad \mathbf{v}_{3}=(-1,-1,1) .
$$

The cone of $\prec_{0} \times \prec_{1}$ is generated by the vectors

$$
\mathbf{v}_{1}=(1,0,0,0), \quad \mathbf{v}_{2}=(-1,1,0,0), \quad \mathbf{v}_{3}=(-1,-1,1,0), \quad \mathbf{v}_{4}=(-1,-1,-1,1),
$$

and $\prec_{0} \times \prec_{1}$ itself is determined by the comparisons $1 \prec 2,12 \prec 3$, and $123 \prec 4$.

The second order, denoted say by $\prec_{2}$, is determined by comparisons $1 \prec 2,2 \prec 3$, and $3 \prec 12$, and its cone is generated by the vectors

$$
\mathbf{u}_{1}=(-1,1,0), \quad \mathbf{u}_{2}=(0,-1,1), \quad \mathbf{u}_{3}=(1,1,-1) .
$$


(Note that the vector $(1,0,0)$ can be obtained from these.) The cone of $\prec_{0} \times \prec_{2}$ is generated by the vectors

$$
\mathbf{u}_{1}=(1,0,0,0), \quad \mathbf{u}_{2}=(-1,-1,1,0), \quad \mathbf{u}_{3}=(-1,0,-1,1), \quad \mathbf{u}_{4}=(-1,1,1,-1),
$$

and $\prec_{0} \times \prec_{2}$ itself is determined by the comparisons $12 \prec 3,13 \prec 4$, and $14 \prec 23$.

Theorem 9. There are 2 orders in $\mathcal{L}_{4}^{*}$ that can be determined by 3 binary comparisons, 11 orders in $\mathcal{L}_{5}^{*}$ that can be determined by 4 comparisons, and 180 orders in $\mathcal{L}_{6}^{*}$ that can be determined by 5 comparisons.

Proof. First, there are two members of $\mathcal{L}_{3}^{*}$. One is the lexicographic order $\preceq_{1}$, which is determined by the two comparisons $1 \prec_{1} 2$ and $12 \prec_{1} 3$, and hence $\preceq_{=} \preceq_{0} \preceq_{1} \in \mathcal{L}_{4}$ is determined by $1 \prec 2,12 \prec 3$ and $123 \prec 4$. The other order $\preceq_{2}$ in $\mathcal{L}_{3}^{*}$ is determined by the three comparisons $1 \prec_{2} 2,2 \prec_{2} 3$ and $3 \prec_{2} 12$, and this cannot be represented as a product of $\preceq_{0}$ and another order, hence $\preceq=\preceq_{0} \times \preceq_{2} \in \mathcal{L}_{4}^{*}$ is determined by $12 \prec 3,13 \prec 4$ and $14 \prec 23$. These are the two orders from Example 7

Next, the 14 members of $\mathcal{L}_{4}^{*}$ are listed in [9]. Here we give the minimal set of determining binary comparisons for each:

1. $\quad 2 \prec 3, \quad 3 \prec 4,4 \prec 12, \quad 14 \prec 23$;

2. $\quad 1 \prec 2,2 \prec 3,4 \prec 12,23 \prec 14$;

3. $\quad 3 \prec 12, \quad 12 \prec 4,4 \prec 13, \quad 14 \prec 23$;

4. $\quad 1 \prec 2, \quad 3 \prec 12, \quad 12 \prec 4,4 \prec 13,23 \prec 14$;

5. $\quad 12 \prec 3, \quad 3 \prec 4,4 \prec 13, \quad 14 \prec 23$;

6. $\quad 1 \prec 2, \quad 12 \prec 3, \quad 4 \prec 13, \quad 23 \prec 14$;

7. $\quad 2 \prec 3,3 \prec 12, \quad 13 \prec 4, \quad 14 \prec 23$;

8. $\quad 2 \prec 3, \quad 3 \prec 12, \quad 13 \prec 4, \quad 4 \prec 23, \quad 23 \prec 14$;

9. $\quad 12 \prec 3, \quad 13 \prec 4, \quad 14 \prec 23$;

10. $\quad 12 \prec 3, \quad 13 \prec 4, \quad 4 \prec 23,23 \prec 14$;

11. $\quad 1 \prec 2,2 \prec 3,3 \prec 12,23 \prec 4,4 \prec 123$;

12. $\quad 1 \prec 2, \quad 2 \prec 3, \quad 3 \prec 12, \quad 123 \prec 4$;

13. $\quad 1 \prec 2, \quad 12 \prec 3, \quad 23 \prec 4, \quad 4 \prec 123$;

14. $\quad 1 \prec 2,12 \prec 3,123 \prec 4$.

In this list, every order determined by 4 comparisons is irreducible, and therefore all of the 11 orders in $\mathcal{L}_{4}^{*}$ that are determined by up to 4 binary comparisons can be extended to orders in $\mathcal{L}_{5}^{*}$ determined by exactly 4 binary comparisons. Finally, the last statement about orders in $\mathcal{L}_{6}^{*}$ follows from our computational results which can be found in [3]. 


\section{A Note on Maclagan's problem}

We know that every representable comparative probability order in $\mathcal{L}_{n}$ corresponds to a region of the hyperplane arrangement $\mathcal{H}_{n}$. A very interesting question, emphasised by Maclagan in [11, p. 295], is this: How many facets do the polytopes associated with the regions of this hyperplane arrangement have?

It is clear that it is sufficient to solve this problem for comparative probability orders in $\mathcal{L}_{n}^{*}$ - that is, those which satisfy (3). We can find a solution computationally in the cases $n=5$ and $n=6$, using the following obvious fact:

Proposition 2. Let $\preceq$ be a representable comparative probability order in $\mathcal{L}_{n}$, and let $P$ be the corresponding convex polytope, which is the closure of a region of the hyperplane arrangement $\mathcal{H}_{n}$. Then the number of significant facets of $P$ equals the number of representable comparative probability orders that are flip-related to $\preceq$.

The results for $n=5$ are given in Tables 1 and 2 below. These numbers are for comparative probability orders from $\mathcal{L}_{5}^{*}$ and $\mathcal{L}_{6}^{*}$, respectively. The total number of comparative probability orders in $\mathcal{P}_{5}$ in each category can be obtained by multiplying by $5 !=120$ and, respectively $6 !=720$.

Table 1: The number of facets of the polytopes associated with $\mathcal{H}_{5}$ corresponding to orders of $\mathcal{P}_{5}^{*}$

\begin{tabular}{|c|c|c|c|c||c|}
\hline \# facets & 5 & 6 & 7 & 8 & all \\
\hline \# regions & 265 & 177 & 65 & 9 & 512 \\
\hline
\end{tabular}

Table 2: The number of facets of the polytopes associated with the regions of $\mathcal{H}_{6}$ corresponding to $\mathcal{L}_{6}^{*}$

\begin{tabular}{|c||c|c|c|c|c|c|c|c||c|}
\hline \# facets & 6 & 7 & 8 & 9 & 10 & 11 & 12 & 13 & all \\
\hline \# orders & 38,025 & 45,536 & 25,931 & 10,280 & 3,400 & 819 & 176 & 20 & 124,187 \\
\hline
\end{tabular}

It is worth paying attention to the fact that for $n=5$ and $n=6$ all comparative probability orders with the largest possible number of flips (namely 8 for $n=5$, and 13 for $n=6$ ) are representable, and all of their flips are friendly. This does not always happen, however, when an order has the smallest possible number of flips.

Maclagan 11] gave an example of a non-representable comparative probability order in $\mathcal{P}_{6}$ whose set of flippable pairs was a subset of the set of all flippable pairs of a representable comparative probability order. She concluded that for $n \geq 6$, an order might not be determined by the set of its flippable pairs.

Strictly speaking, we have to talk about the sequence of flippable pairs of an order $\preceq$, since these pairs may occur in $\preceq$ in a different order. Strengthening the result of Maclagan, we have found eight sequences of comparisons with the property that each is the sequence of flippable pairs for two different 
non-representable comparative probability orders in $\mathcal{P}_{6}$. We list these eight sequences below:

$$
\begin{aligned}
& (14 \prec 5, \quad 15 \prec 24, \quad 125 \prec 34, \quad 45 \prec 16, \quad 26 \prec 145, \quad 1245 \prec 36) \text {; } \\
& (14 \prec 5, \quad 15 \prec 24, \quad 125 \prec 34, \quad 45 \prec 16, \quad 26 \prec 14, \quad 36 \prec 1245) \text { ); } \\
& (5 \prec 14, \quad 24 \prec 15, \quad 125 \prec 34, \quad 16 \prec 45, \quad 145 \prec 26, \quad 1245 \prec 36) \text {; } \\
& (5 \prec 14, \quad 24 \prec 15, \quad 34 \prec 125, \quad 16 \prec 45, \quad 145 \prec 26, \quad 1245 \prec 36) \text {; } \\
& (4 \prec 13, \quad 23 \prec 14, \quad 125 \prec 34, \quad 134 \prec 6, \quad 16 \prec 234, \quad 1245 \prec 36 \text { ); } \\
& (4 \prec 13, \quad 23 \prec 14, \quad 125 \prec 34, \quad 134 \prec 6, \quad 16 \prec 234, \quad 36 \prec 1245) \text { ); } \\
& (13 \prec 4, \quad 14 \prec 23, \quad 125 \prec 34, \quad 6 \prec 134, \quad 234 \prec 16, \quad 1245 \prec 36) \text {; } \\
& (13 \prec 4, \quad 14 \prec 23, \quad 34 \prec 125, \quad 6 \prec 134, \quad 234 \prec 16, \quad 1245 \prec 36) \text {. }
\end{aligned}
$$

These were found with the help of the MAGMA [1] system, which we used to determine and analyse several examples of orders on sets of small order. More computational results on the flip relation for $n=5$ and $n=6$ can be found in 3 .

\section{$9 \quad$ Further research}

A number of interesting questions remain open. Among those are the following:

- Is the graph $\mathcal{P}_{n}$ connected? (See [11, p. 295])

- Can any order in $\mathcal{P}_{n}$ be connected to a representable order by a sequence of non-central flips?

- What is a sharp lower bound for the number of flippable pairs of a non-representable comparative probability order? At the moment we can guarantee only one flippable pair (viz. the central one).

- What is a sharp upper bound on the number of flippable pairs for orders in $\mathcal{L}_{n}$ ? and in $\mathcal{P}_{n}$ ?

- Are all of the comparative probability orders in $\mathcal{P}_{n}$ having the maximum possible number of flippable pairs representable? Are all their neighbors representable?

\section{References}

[1] Bosma, W., Cannon, J., and Playoust, C. (1997). The Magma Algebra System I: The User Language, J. Symbolic Comput. 24, 235-265.

[2] Christian, R. and Slinko, A. (2005). Answers to Two Questions of Fishburn on Subset Comparisons in Comparative Probability Orderings, Proceedings of The 4th International Symposium on Imprecise Probabilities and Their Applications (ISIPTA 05), Pittsburg, Pensylvania, 2005, 117-124.

[3] Christian, R., Conder, M. and Slinko, A. (2006). Flippable Pairs and Subset Comparisons in Comparative Probability Orderings and Related Simple Games, The Centre for Interuniversity Research in Qualitative Economics (CIREQ), Cahier 15-2006. University of Montreal, 2006 http://www.cireq.umontreal.ca/publications/15-2006-cah.pdf 
[4] Conder, M. and Slinko, A. (2004). A counterexample to Fishburn's conjecture on finite linear qualitative probability. Journal of Mathematical Psychology 48, 425-431.

[5] Fine, T., and Gill, J., (1976) The enumeration of comparative probability relations. Annals of Probability 4: 667-673.

[6] de Finetti, B. (1931). Sul significato soggetivo della probabilità, Fundamenta Mathematicae 17, $298-329$.

[7] Fishburn, P.C. (1996). Finite Linear Qualitative Probability, Journal of Mathematical Psychology 40, 64-77.

[8] Fishburn, P.C. (1997). Failure of Cancellation Conditions for Additive Linear Orders, Journal of Combinatorial Designs 5, 353-365.

[9] Fishburn, P.C., Pekeč A., Reeds, J.A. (2002). Subset Comparisons for Additive Linear Orders, Mathematics of Operations Research 27, 227-243.

[10] Kraft, C.H., Pratt, J.W., and Seidenberg, A. (1959). Intuitive Probability on Finite Sets, Annals of Mathematical Statistics 30, 408-419.

[11] Maclagan D. (1999) Boolean Term Orders and the Root System $B_{n}$. Order 15, 279-295.

[12] Orlik, P., and Terao, H. (1992) Arrangements of Hyperplanes. Springer-Verlag, Berlin.

[13] Scott, D. (1964) Measurement structures and inequalities, Journal of Mathematical Psychology 1, 233-247.

[14] Stanley, R. (2004) An Introduction to Hyperplane Arrangements. Lecture Notes for Graduate Students. MIT. http://www-math.mit.edu/ ${ }_{\text {rstan/arrangements/arr.html }}$ 